\title{
Knockdown of mediator complex subunit 19 inhibits the growth of ovarian cancer
}

\author{
YINGTAO LIU ${ }^{1,3}$, XIANG TAO $^{1}$, LINGLING FAN ${ }^{1}$, LUOQI JIA ${ }^{1}$, CHAO GU $^{1}$ and YOUJI FENG ${ }^{1,2}$ \\ ${ }^{1}$ Department of Gynecology, Obstetrics and Gynecology Hospital of Fudan University, Shanghai 200011; \\ ${ }^{2}$ Department of Obstetrics and Gynecology, Shanghai First People's Hospital, Jiao Tong University, Shanghai 200080; \\ ${ }^{3}$ Shanghai Key Laboratory of Female Reproductive Endocrine Related Diseases, Shanghai 200011, P.R. China
}

Received April 30, 2012; Accepted August 24, 2012

DOI: $10.3892 / \mathrm{mmr} .2012 .1065$

\begin{abstract}
Ovarian cancer causes more deaths than any other type of female reproductive cancer. The development of new therapeutic approaches is required due to the low survival rate using routine methods. The goal of this study was to investigate the effect of the gene silencing of mediator complex subunit 19 (MED19) on cell viability and tumor growth in ovarian cancer. Immunohistochemistry was used to characterize the expression of MED19 in human ovarian cancer tissues. Lentivirus-mediated RNAi was employed to downregulate endogenous MED19 expression in SKOV-3 and HEY ovarian cancer cells. MTT assay, BrdU incorporation assay, colony formation assay, cell cycle analysis and tumor xenografts in nude mice were performed to determine the effects of MED19 silencing on cell viability and tumor growth in vitro and in vivo. The data showed that the expression of MED19 in human ovarian cancer tissues correlated with the level of tumor malignancy. The downregulation of MED19 in ovarian cancer cells significantly inhibited cell proliferation and colony formation in vitro and led to cell cycle arrest in the G0/G1 phase. MED19 RNAi significantly inhibited ovarian cancer tumor growth in engrafted nude mice. Our findings reveal that the knockdown of MED19 by lentivirus-mediated RNAi may be useful in the treatment of human ovarian cancer.
\end{abstract}

\section{Introduction}

The management of ovarian cancer is a major challenge for gynecological oncologists. Since the symptoms of ovarian cancer are non-specific, more than $2 / 3$ of the cases are diagnosed at advanced stages $(1,2)$. Debulking surgery followed by chemotherapy continues to be the standard treatment for

Correspondence to: Professor Youji Feng, Department of Gynecology, Obstetrics and Gynecology Hospital of Fudan University, 419 Fang Xie Road, Shanghai 200011, P.R. China

E-mail: rab25.fsh@hotmail.com

Key words: mediator complex subunit 19, ovarian cancer, proliferation, lentivirus advanced ovarian cancer (3). Despite a high response to the initial therapy, many of the patients relapse eventually and succumb to chemotherapy-resistant diseases. As a result, the overall 5-year survival rate of advanced ovarian cancer remains low, at approximately $30 \%(1,4)$. The poor outcome of ovarian cancer necessitates our efforts towards better understanding its biological behavior and identifying new prognostic and therapeutic targets.

The mediator complex subunit 19 (MED19) was originally recognized in a search for mutants with increased aerobic expression of the CYC7 gene $(5,6)$. The mediator complex is a multiprotein transcriptional co-activator that is expressed ubiquitously in eukaryotes and is required for the induction of RNA polymerase II transcription (7-9). A series of mediator complexes has been identified in mammals by multidimensional protein identification technology. These complexes include the thyroid hormone receptor-associated protein/ SRB-Med-containing co-factor (TRAP/SMCC), the activatorrecruited factor-large (ARC-L), vitamin D receptor-interacting protein (DRIP), mouse mediator, positive co-factor 2 (PC2) and the co-factor required for $\mathrm{Sp} 1$ transcriptional activation (CRSP) complexes (10).

The aberrant transcription of genes is believed to be one of the causes of human cancer. The silencing of MED19 may interfere with the transcriptional process and has been demonstrated to inhibit the growth of pancreatic cancer (11). The role of MED19 in ovarian cancer, however, has never been reported. The goal of this study was to investigate the effect of MED19 gene silencing on cell viability and tumor growth in ovarian cancer. Our results demonstrated that MED19 expression is correlated with malignancy and histological grading of human ovarian tumors. The knockdown of MED19 with lentivirus-mediated RNAi reduced the viability of ovarian cancer cells in vitro and inhibited tumor growth in xenografted nude mice. These data suggest that MED19 may serve as a potential target in the treatment of ovarian cancer.

\section{Materials and methods}

Cell lines. The SKOV-3 and HEY human ovarian cancer cell lines were purchased from ATCC (Manassas, VA, USA). The cells were maintained in RPMI-1640 medium (Gibco, Grand Island, NY, USA) containing 10\% fetal bovine serum (FBS), 
$100 \mathrm{U} / \mathrm{ml}$ penicillin and $100 \mu \mathrm{g} / \mathrm{ml}$ streptomycin and were cultured in a humidified atmosphere of $5 \% \mathrm{CO}_{2}$ at $37^{\circ} \mathrm{C}$.

Tissue microarray and immunohistochemistry. Tissue microarrays of ovarian disease (OV1005) were obtained from Alenabio (Xi'an, China), and contained 18 ovarian cystadenoma, 7 varian borderline cystadenoma and 52 ovarian carcinoma tissue cores with 24 stage I, 9 stage II, 18 stage III and 1 stage IV ovarian cancer samples.

Tissue microarray sections were stained with rabbit antiMED19 antibody (1:250, Abcam Inc., Cambridge, MA, USA) using a goat anti-rabbit ABC immunohistochemistry kit (Mingrui Inc., Shanghai, China). The immunostaining patterns for MED19 were assessed by Image-Pro Plus software (Media Cybernetics, Silver Spring, MD, USA).

Construction of lentiviral vectors. To generate lentivirus expressing RNAi specific for the MED19 gene, the RNA interference sequence for human MED19 (aaGGTGAAGGAGA AGCTAAGT) was designed with the manufacturer's RNAi Designer program, and the negative control construct was created with a scrambled sequence (TTCTCCGAACGTGTC ACGT). The segments of nucleotides were cloned into the $H p a \mathrm{I}$ and $\mathrm{XhoI}$ sites of the pGCSIL-GFP vector (GeneChem, Shanghai, China) to generate pGCSIL-GFP-MED19 (siMed) and pGCSIL-GFP-nonsense (siNon).

Lentivirus transduction. Cells were plated at $40-50 \%$ confluence and incubated at $37^{\circ} \mathrm{C}$. After $24 \mathrm{~h}$ of incubation, cells were infected with recombinant lentiviral vectors at a multiplicity of infection of 40 and then incubated at $37^{\circ} \mathrm{C}$ for $10 \mathrm{~h}$. The viral supernatant was then replaced with fresh medium. At day 5 post-transduction, the knockdown efficiency of RNAi was determined by quantitative real-time RT-PCR and western blot analysis.

$R N A$ extraction and quantitative real-time RT-PCR. Total RNA was extracted from SKOV-3 cells with TRIzol reagent (Invitrogen, Carlsbad, CA, USA). The RNA expression levels of MED19 were detected by quantitative real-time RT-PCR using the SYBR-Green RT-PCR kit (Takara) according to the manufacturer's instructions. Briefly, the cDNA was synthesized using the RevertAid First-Strand cDNA Synthesis kit (Fermentas, Lithuania). The cDNA was used as the template and was subjected in triplicate to denaturation at $94^{\circ} \mathrm{C}$ for $1 \mathrm{~min}$ and 40 cycles at $94^{\circ} \mathrm{C}$ for $5 \mathrm{sec}$ and at $60^{\circ} \mathrm{C}$ for $31 \mathrm{sec}$ in an ABI PRISM 7000 Sequence Detection System (Applied Biosystems, Foster City, CA, USA). The specific primer pairs were as follows: MED19 (107 bp) sense, 5'-TGACAGGCAGCACGAATC-3' and antisense, 5'-CAGGTCAGGCAGGAAGTTAC-3'; $\beta$-actin (202 bp) sense, 5'-GGCGGCACCACCATGTACCCT-3' and antisense, 5'-AGGGGCCGGACTCGTCATACT-3'. The fold change in the expression of MED19 was calculated using the $-\Delta \Delta \mathrm{Ct}$ method, with $\beta$-actin as the internal control.

Western blot analysis. The cultured cells were washed with phosphate-buffered saline and then lysed with RIPA solution (Bocai, Shanghai, China). The lysate was cleared by centrifugation at 12,000 rpm for $30 \mathrm{~min}$ and the protein was then quantified using the bicinchoninic acid (BCA) method
(Bocai) according to the manufacturer's instructions. A total of $30 \mu \mathrm{g} /$ lane proteins were resolved by SDS-PAGE and transferred onto a PVDF membrane (Millipore, Billerica, MA, USA). After blocking with 5\% BSA-TBST for $1 \mathrm{~h}$ at room temperature, the membrane was incubated with anti-MED19 (1:250, Abcam Inc.) and anti-GAPDH antibody (1:5,000; Kangchen, Shanghai, China) overnight at $4^{\circ} \mathrm{C}$. The membrane was then incubated with HRP-conjugated secondary antibodies (Kangchen) $(1: 5,000)$ for $1 \mathrm{~h}$ at $37^{\circ} \mathrm{C}$. Chemiluminescent detection was performed using the ECL kit (Perkin-Elmer, Waltham, MA, USA).

MTT assay. Cell viability and proliferation were evaluated using a modified 3-(4,5-dimethyldiazol-2-yl)-2,5-diphenylte trazolium bromide (MTT) assay. Cells in exponential growth were plated at a final concentration of $4 \times 10^{3}$ cells/well in 96-well culture plates and were harvested at 1, 2, 3, 4 and 5 days later accordingly. A total of $20 \mu \mathrm{l}$ of MTT $(5 \mathrm{mg} / \mathrm{ml})$ (Sigma-Aldrich, St. Louis, MO, USA) was added to the cells. After incubation for an additional $4 \mathrm{~h}$, the reaction was stopped by removal of the supernatant and $100 \mu 1$ DMSO was added to dissolve the formazan product. After $30 \mathrm{~min}$, the optical density (OD) of each well was measured at $570 \mathrm{~nm}$ using an ELISA reader.

BrdU incorporation assay. DNA synthesis in the proliferating cells was determined by measuring the BrdU incorporation. SKOV-3 and HEY cells transduced with siMed or siNon were seeded in 96-well culture plates at a density of $4 \times 10^{3}$ cells/well and cultured for 24 or $48 \mathrm{~h}$. The cells were then incubated with BrdU (BD Pharmingen, San Diego, CA, USA) at a final concentration of $10 \mu \mathrm{M}$ for $2-24 \mathrm{~h}$. The medium was removed at the end of incubation, and the cells were fixed with $70 \%$ alcohol and labeled with a peroxidase-conjugated anti-BrdU antibody (Sigma-Aldrich). After incubation for $60 \mathrm{~min}$, the cells were washed 3 times with phosphate-buffered saline. Tetramethylbenzidine was added thereafter as the peroxidase substrate for $30 \mathrm{~min}$ and the absorbance values were measured at $490 \mathrm{~nm}$ using an ELISA reader.

Colony formation assay. The effect of MED19 gene silencing on colony formation in SKOV-3 cells was analyzed by colony formation assays. Cells $\left(3 \times 10^{2}\right)$ were plated in $10-\mathrm{cm}$ culture dishes and cultured in 10\% FBS RPMI-1640 and 5\% $\mathrm{CO}_{2}$ at $37^{\circ} \mathrm{C}$ for 2 weeks. The cell colonies were washed twice with phosphate-buffered saline and fixed by $4 \%$ PFA for $15 \mathrm{~min}$. Cells were then stained with Giemsa for 20 min and washed twice with $\mathrm{ddH}_{2} \mathrm{O}$. Visible colonies were manually counted.

Cell cycle analysis by flow cytometry. Cellular DNA content was detected with a flow cytometer (BD, Franklin Lakes, NJ, USA), in which $5 \times 10^{4}$ events were collected. The list mode data were regrouped into DNA histograms, and individual cell cycle phase fractions were quantified using ModFit 3.0 analysis software (Verity Software Inc., Topsham, ME, USA).

Tumor xenografts in nude mice. Female BALB/c mice were treated according to the protocol approved by the Ethics Committee of Fudan University. Six-week-old mice ( $n=4$ per group) were injected subcutaneously either with parent SKOV-3 

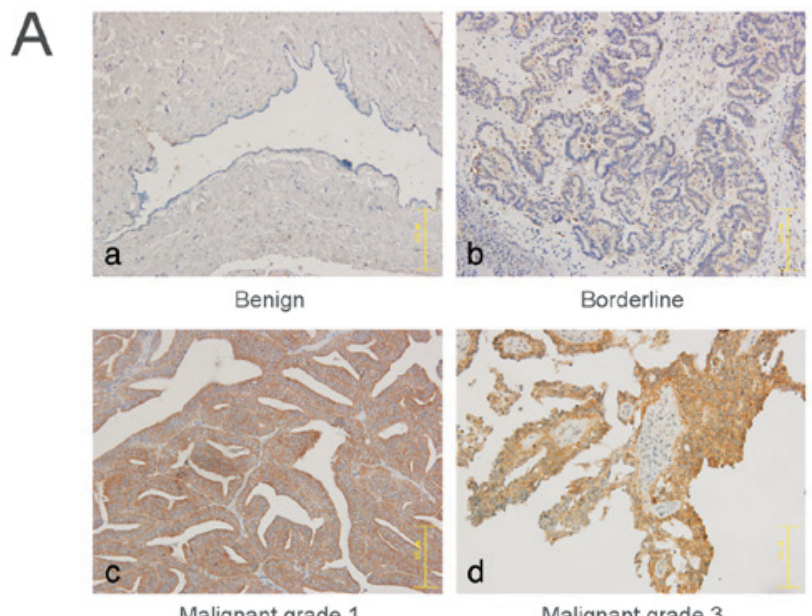

Malignant grade 1

Malignant grade 3
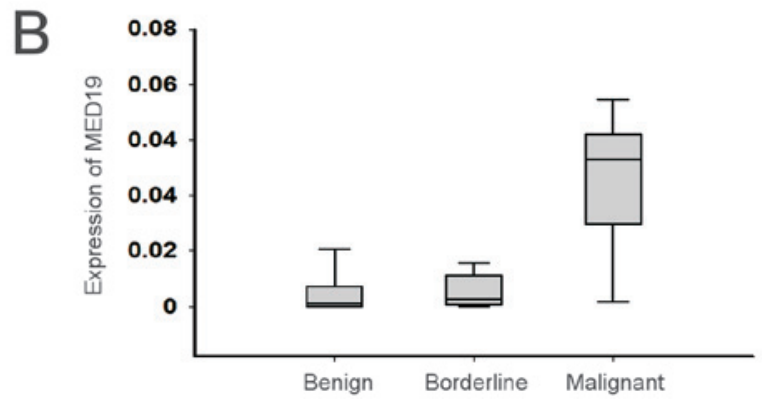

Figure 1. Expression levels of MED19 in ovarian tumor tissues. (A) Immunohistochemical staining of ovarian tumor tissue microarray with MED19. Representative slides of (a) cystadenoma, (b) borderline cystadenoma (c) grade 1 endometrioid adenocarcinoma and (d) grade 3 serous adenocarcinoma. (B) Expression levels of MED19 in benign, borderline and malignant ovarian tumor tissues.

cells, siNon-transduced SKOV-3 cells or siMed-transduced SKOV-3 cells $\left(1 \times 10^{7}\right.$ cells in $200 \mu$ l RPMI-1640) in the right forelimbs. The mice were sacrificed at day 42 after injection. Tumor size was measured with calipers weekly and tumor volume was calculated using the following formula: tumor volume $\left(\mathrm{mm}^{3}\right)=$ tumor length $(\mathrm{mm}) \mathrm{x}$ tumor width $(\mathrm{mm}) / 2$. Bodyweight was also measured to assess any side-effects.

Statistical analysis. Immunohistochemistry data were analyzed by one-way ANOVA using SPSS software (Release 15.0,
SPSS Inc.). Data from the in vitro studies were expressed as the means \pm SE. The difference between the 2 groups was analyzed by one-way ANOVA followed by Dunnett's test. $\mathrm{P}<0.05$ was considered to indicate a statistically significant difference. Representative images from microscopy and western blot analysis are shown.

\section{Results}

Expression levels of MED19 in benign, borderline and malignant ovarian tumor tissue. MED19 expression in ovarian tumor tissue was examined by immunohistochemistry in a tissue microarray that contained ovarian cystadenoma, borderline cystadenoma and carcinoma samples. Following the incubation of the tissue microarrays with anti-MED19 antibody, the staining was mainly confined to the tumor tissue, whereas the majority of the mesenchymal tissue was unstained (Fig. 1A). The mean MED19 expression was significantly lower in the benign $(0.008 \pm 0.009)$ or borderline ovarian cystadenomas $(0.006 \pm 0.006)$ compared to the ovarian carcinomas $(0.054 \pm 0.030)(\mathrm{P}<0.01)($ Fig. 1B). Among the ovarian carcinoma tissues, MED19 expression was higher in stage II and III than in stage I tumors $(\mathrm{P}<0.05)$. MED19 expression in the ovarian carcinoma tissues, however, did not correlate with age, tumor stage or histological type $(\mathrm{P}>0.05)$ (Table I).

Knockdown of MED19 with lentiviral transduction in ovarian cancer cells. The SKOV-3 and HEY human ovarian cancer cells were infected with lentivirus that contained either siMed or siNon. Efficient transduction was confirmed by GFP expression in cells observed under a fluorescent microscope (Fig. 2A). Twenty-four hours after transduction, the MED19 mRNA expression was downregulated by $84 \%$ in the cells transduced with siMed compared to those transduced with siNon $(\mathrm{P}<0.01)$ (Fig. 2B). A similar result was noted in western blot analysis which showed that MED19 protein expression was downregulated in the SKOV-3 cells following siMed transduction (Fig. 2C).

Knockdown of MED19 inhibits the proliferation of ovarian cancer cells. The proliferation dynamics of the parental, siNon-transduced and siMed-transduced cells were determined by MTT assays. Cell proliferation was monitored for
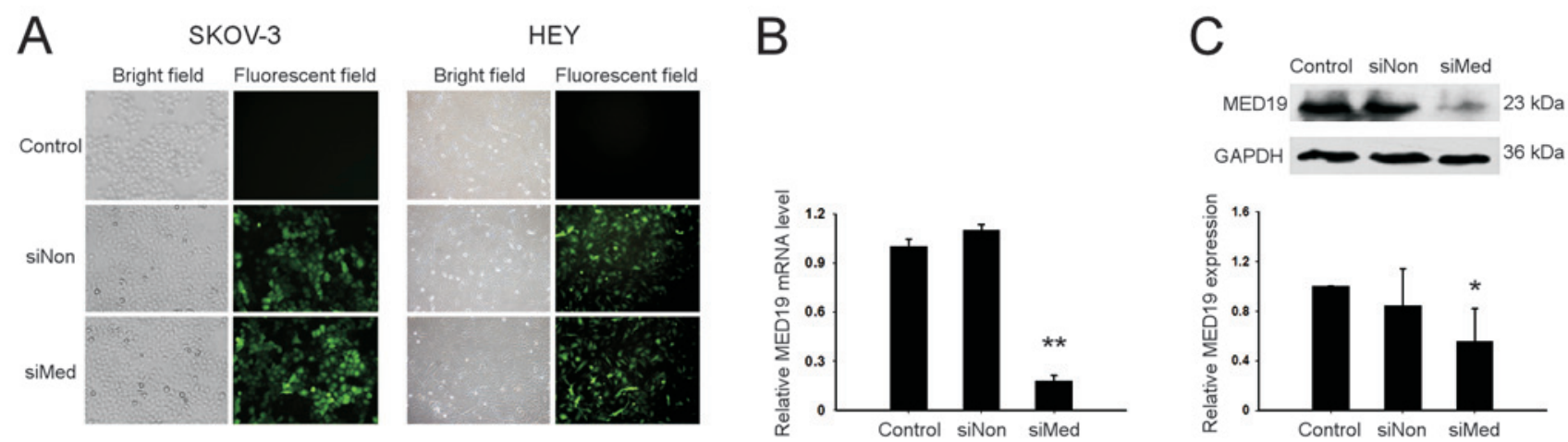

Figure 2. Downregulation of MED19 expression in ovarian cancer cells by lentivirus-mediated RNAi. (A) GFP expressed after the transduction of SKOV-3 and HEY cells with siNon and siMed. (B) MED19 mRNA levels were detected by real-time RT-PCR. Data are expressed as fold changes of MED19 mRNA transcripts. Data shown are the means \pm SEM of a representative experiment performed in triplicate, ${ }^{* *} \mathrm{P}<0.01$. (C) MED19 protein levels were detected by western blot analysis. ${ }^{*} \mathrm{P}<0.05$. 
A

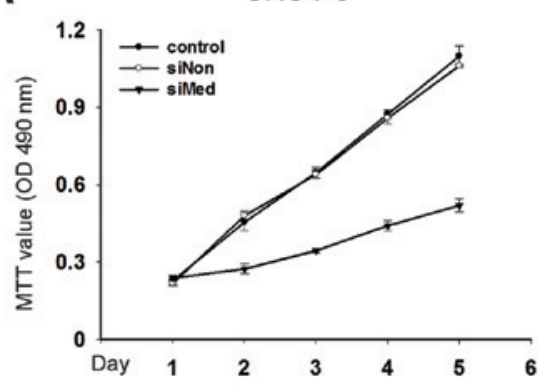

C

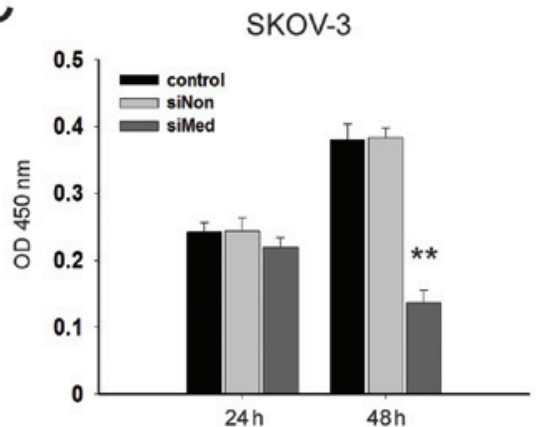

E
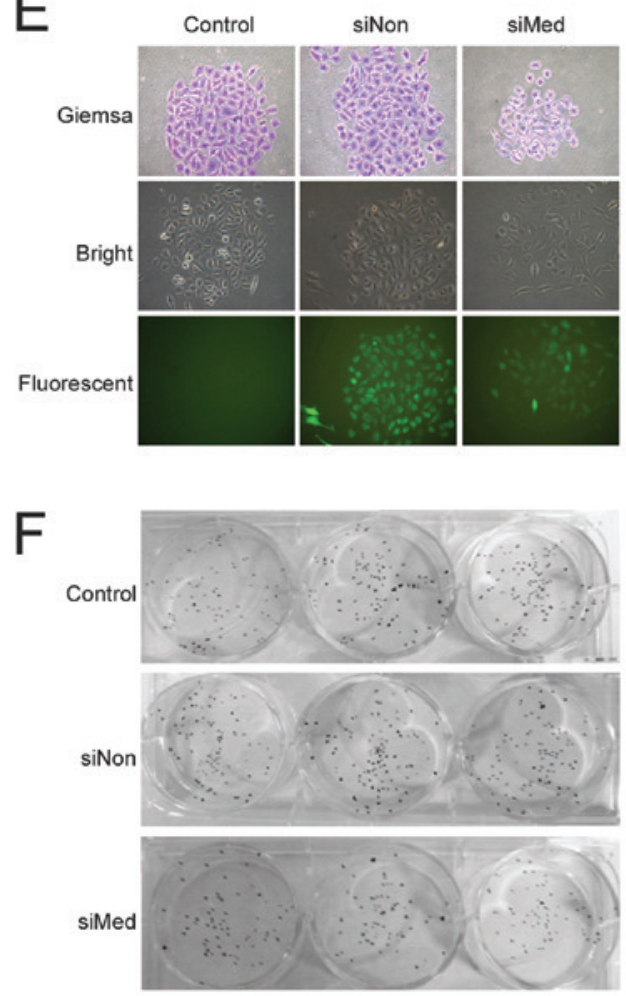

B

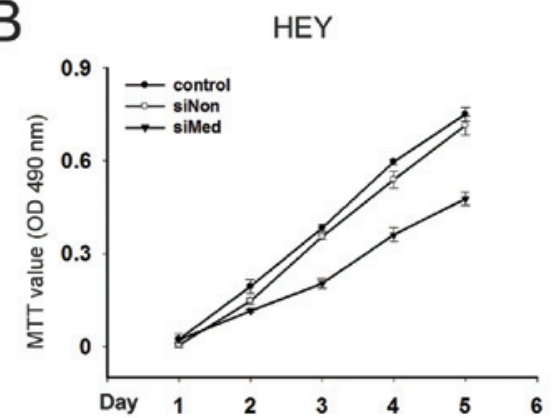

D

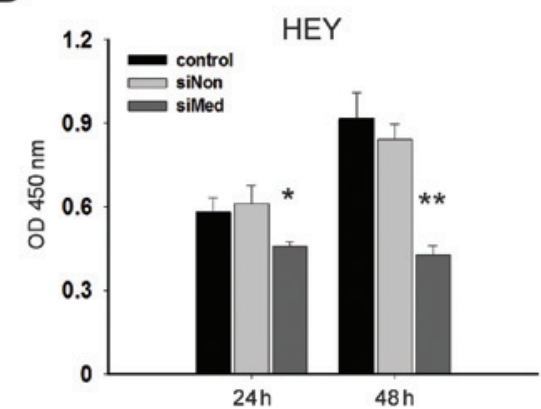

SKOV-3

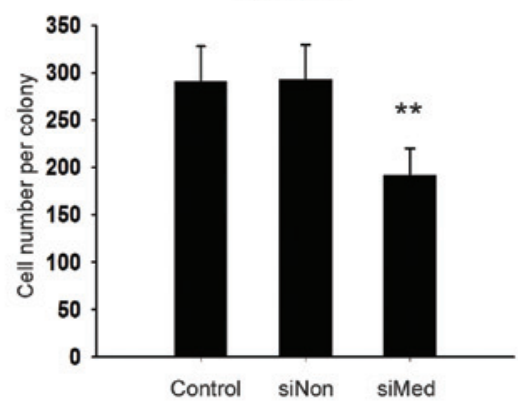

SKOV-3

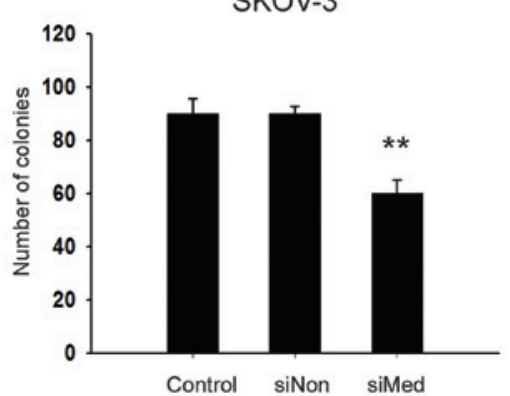

Figure 3. Proliferation and colony formation ability in ovarian cancer cells after MED19 knockdown. (A and B) The growth of SKOV-3 and HEY cells was assessed by MTT assay on days 1, 2,3,4 and 5 in parental cells and cells transduced with siNon or siMed. (C and D) Levels of BrdU incorporation $48 \mathrm{~h}$ after siNon or siMed transduction. (E) Colony formation assay showed that siMed-infected SKOV-3 cells have fewer cells in each colony compared to the siNon group. (F) Colony formation assay showed that siMed-infected SKOV-3 cells produced fewer colonies compared with the siNon group. " $\mathrm{P}<0.05$; ** $\mathrm{P}<0.01$.

5 consecutive days following lentiviral infection. The results showed that the growth of SKOV-3 and HEY cells transduced with siMed was significantly inhibited compared with the siNon-transduced and parental cells (Fig. 3A and B). A 70\% reduction in viability was noted in the SKOV-3 cells with
MED19 gene silencing compared with the siNon group on day 5 .

To further investigate the effects of MED19 gene silencing on DNA synthesis in ovarian cancer cells, a BrdU incorporation assay was performed. Following treatment with siMed for 
A

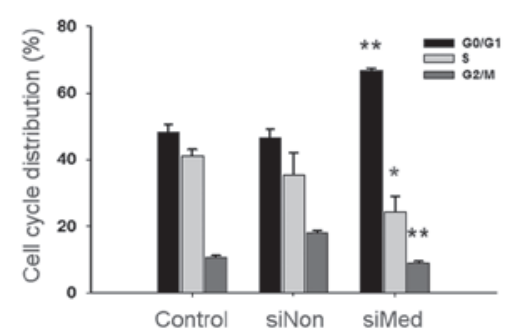

B

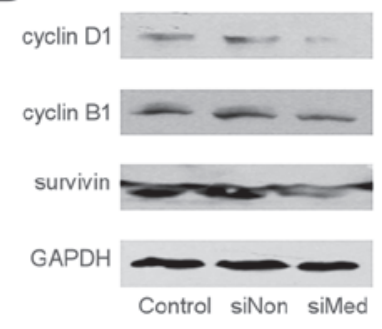

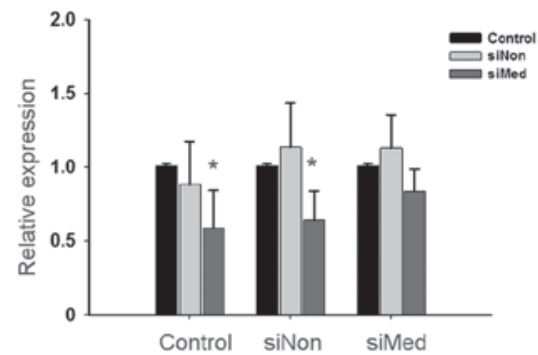

Figure 4. Cell cycle analysis in ovarian cancer cells following MED19 knockdown. (A) Parental, siNon and siMed cells were fixed with 70\% ethanol and stained with propidium iodide and analyzed by fluorescence-activated cell sorting. The relative frequency of each phase of the cell cycle was expressed as the mean percentage from 3 independent experiments. ${ }^{*} \mathrm{P}<0.05 ;{ }^{* *} \mathrm{P}<0.01$. (B) The expression levels of cyclin D1, cyclin B1 and survivin in parental, siMed and siNon cells analyzed by western blot analysis. ${ }^{*} \mathrm{P}<0.05$.

Table I. Expression levels of MED19 in ovarian cancer and clinical factors.

\begin{tabular}{lrcr}
\hline Clinical factors & $\mathrm{N}$ & MED19 expression & P-value \\
\hline $\begin{array}{l}\text { Age (years) } \\
\quad 555\end{array}$ & 31 & $0.057 \pm 0.033$ & \\
$\quad \geq 55$ & 23 & $0.051 \pm 0.025$ & 0.472 \\
Tumor stage & & & \\
$\quad$ I, II & 35 & $0.054 \pm 0.031$ & \\
$\quad$ III, IV & 19 & $0.057 \pm 0.030$ & 0.821 \\
Pathological grade & & & \\
$\quad$ Grade 1 & 10 & $0.041 \pm 0.025$ & 0.047 \\
$\quad$ Grades 2 and 3 & 40 & $0.059 \pm 0.031$ & \\
Histological type & & & \\
$\quad$ Serous & 36 & $0.057 \pm 0.028$ & \\
$\quad$ Mucinous & 4 & $0.049 \pm 0.032$ & \\
$\quad$ Endometroid & 14 & $0.050 \pm 0.038$ & \\
\hline
\end{tabular}

$24 \mathrm{~h}$, the rate of DNA synthesis was not significantly affected in the SKOV-3 and HEY cells. After $48 \mathrm{~h}$ of transduction with siMed, however, the DNA synthesis was suppressed by $39 \%$ in the SKOV-3 cells and $41 \%$ in the HEY cells compared to the cells that were transduced with siNon (Fig. 3C and D).

As the HEY cells were not capable of forming colonies in this study, SKOV-3 cells were used to examine the effects of MED19 gene silencing on colony forming potential. The results showed that the siMed-transduced SKOV-3 cells formed fewer cells in each colony and $33 \%$ fewer colonies compared to the siNon-transduced cells $(\mathrm{P}<0.05)$ (Fig. $3 \mathrm{E}$ and $\mathrm{F})$.

Knockdown of MED19 induces cell cycle arrest in ovarian cancer cells. Cellular DNA contents of parental, siNon-transduced and siMed-transduced cells were analyzed by fluorescence-activated cell sorting to determine their cell cycle status. The results demonstrated that the siMed-transduced SKOV-3 and HEY cells had a higher percentage of cells in the G0/G1 phase (66.8 vs. $46.6 \%$ in SKOV-3 cells and 73.9 vs. $68.8 \%$ in HEY cells) and a lower percentage of cells progressing into the S phase (24.4 vs. $35.4 \%$ in SKOV-3 cells and 17.3 vs. $22.8 \%$ in HEY cells) compared to the siNon-transduced cells (Fig. 4A).

Expression of cyclin D1, cyclin B1 and survivin detected by western blot analysis to verify cell cycle status. The transduction of SKOV-3 and HEY cells with siMed decreased the expression levels of cyclin D1 and cyclin B1. The expression level of survivin was also downregulated by MED19 gene silencing (Fig. 4B), which indicated that the knockdown of MED19 arrested the cell cycle progression of ovarian cancer cells.

Knockdown of MED19 inhibits the growth of ovarian cancer in vivo. The effect of MED19 gene silencing on the growth of ovarian cancer was analyzed in nude mice that were injected with siMed-transduced cells. Compared with the mice that were injected with parental and siNon-transduced cells, tumor growth was significantly delayed in the siMed group, which was evident from 2 weeks after transplantation until the day when the mice were sacrificed (Fig. 5). At day 42, the xenograft tumor weights were $372.5 \pm 277.6 \mathrm{mg}$ in the mice injected with parental cells, $260.0 \pm 126.6 \mathrm{mg}$ in the siNon group and $12.5 \pm 2.5 \mathrm{mg}$ in the siMed group. No marked change in behavior, appearance or body weight was observed in the studied mice.

\section{Discussion}

Ovarian cancer is one of the most lethal gynecological malignancies. Although new chemotherapeutic agents have improved the survival rate for ovarian cancer patients over the past few decades, overall mortality from the disease still remains high. For patients in stage III and IV, the 5-year survival is approximately 34 and $18 \%$, respectively (13-16). The reduced 5-year survival rates necessitate the need to develop an improved understanding of cell growth and death in ovarian cancer to identify new etiologic, prognostic or therapeutic targets.

MED19 is a component of the mediator complex, which is a co-activator for DNA binding factors that activate transcription via RNA polymerase II. Mediator is recruited to promoters by direct interactions with regulatory proteins and serves as a scaffold for the assembly of a functional pre-initiation 
A

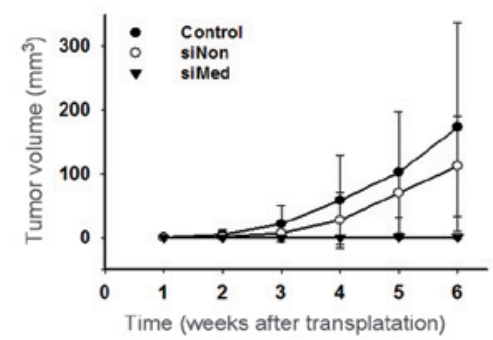

B

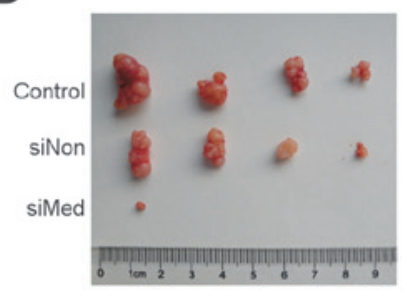

C

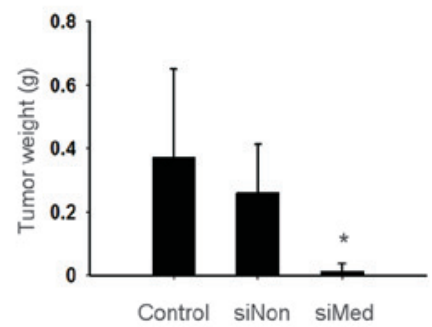

Figure 5. MED19 knockdown inhibited the growth of ovarian cancer in vivo. Nude mice were injected with parental, siNon- and siMed-infected cells. (A) Growth curves of ovarian tumors. (B) Tumor xenografts of mice bearing tumors at the study endpoint. (C) Tumor weight at the end of the study. Values represent the means \pm SEM. ${ }^{*} \mathrm{P}<0.05$ compared with the siNon group.

complex with RNA polymerase II and general transcription factors. In this capacity, MED19 plays an essential role in regulating the assembly and/or activity of RNA polymerase II transcription complexes on core promoters (12). Though MED19 gene silencing has been reported to inhibit the growth of pancreatic cancer, the role of MED19 in ovarian cancer remains unknown. Therefore, we genetically downregulated the expression of MED19 in the SKOV-3 and HEY human ovarian cancer cell lines by infecting them with MED19specific RNAi-expressing lentivirus and determined the role of MED19 in the proliferation and growth of tumor cells. We found that the downregulation of endogenous MED19 expression inhibited the growth, decreased the proliferation and led to cell cycle arrest in ovarian cancer cells. To the best of our knowledge, this is the first study to show that the mediator complex is important in ovarian cancer cell growth and proliferation.

The expression of MED19 correlated with the progression of ovarian carcinoma, suggesting the relevance of MED19 to the tumorigenesis of ovarian cancer. The higher MED19 expression was associated with a poorer differentiation, indicating that MED19 is also involved in the regulation of differentiation. Due to the lack of follow-up data, we were unable to determine whether a correlation exists between the survival rates and MED19 expression in ovarian cancer.

Gene therapy, an applied form of biotechnology, is a new, innovative approach to the treatment of human cancer (17-20). The therapeutic efficiency of cancer gene therapy strategies strongly depends on the efficiency of gene transfer. Lentivirus, a member of slow viruses of the Retroviridae family, has been characterized as having a long incubation period. Lentivirus is capable of delivering a large amount of genetic information into the host cell and is one of the most efficient methods of gene delivery. Several studies have focused on evaluating the therapeutic effects of gene silencing in a variety of diseases, particularly since antisense technology allows for the highly specific and effective downregulation of target gene expression (17-20). RNAi is a useful tool for the functional analysis of genes and may be a potential therapeutic strategy for various diseases, including cancers. Thus, the combination of lentivirus and RNAi may provide a novel therapeutic strategy for cancer gene therapy.

Our study using the 2 human ovarian cancer cell lines, SKOV-3 and HEY, showed that the lentivirus-mediated downregulation of MED19 decreased cell proliferation and growth. Therefore, a new strategy via the reduction of MED19 expression mediated by lentivirus may be useful in the treatment of human ovarian cancer.

Many other mediator complexes exist in the mediator gene family. Whether other members of the mediator complex are abnormally regulated in human ovarian cancer or other human cancers remains unknown. The effectiveness of MED19 RNAi therapy in human ovarian cancer and other human cancer warrants further investigation.

\section{Acknowledgements}

This research was sponsored by the Fundamental Research Funds for the Central Universities of Fudan University.

\section{References}

1. Colombo N, van Gorp T, Parma G, et al: Ovarian cancer. Crit Rev Oncol Hemat 60: 159-179, 2006.

2. Jacobs IJ and Menon U: Progress and challenges in screening for early detection of ovarian cancer. Mol Cell Proteomics 3: 355-366, 2004.

3. Armstrong DK, Bundy B, Wenzel L, et al: Intraperitoneal cisplatin and paclitaxel in ovarian cancer. N Engl J Med 354: 34-43, 2006.

4. Jemal A, Siegel R, Ward E, et al: Cancer statistics 2007. CA Cancer J Clin 57: 43-66, 2007.

5. Rosenblum-Vos LS, Rhodes L, Evangelista CC, et al: The ROX3 gene encodes an essential nuclear protein involved in CYC7 gene expression in Saccharomyces cerevisiae. Mol Cell Biol 11: 5639-5647, 1991

6. Gustafsson CM, Myers LC, Li Y, et al: Identification of Rox3 as a component of mediator and RNA polymerase II holoenzyme. J Biol Chem 272: 48-50, 1997.

7. Jiang YW, Veschambre P, Erdjument-Bromage $\mathrm{H}$, et al: Mammalian mediator of transcriptional regulation and its possible role as an end-point of signal transduction pathways. Proc Natl Acad Sci USA 95: 8538-8543, 1998.

8. Kornberg RD: Mediator and the mechanism of transcriptional activation. Trends Biochem Sci 30: 235-239, 2005.

9. Kim YJ, Bjorklund S, Li Y, et al: A multiprotein mediator of transcriptional activation and its interaction with the c-terminal repeat domain of RNA-polymerase II. Cell 77: 599-608, 1994.

10. Sato S, Tomomori-Sato C, Parmely TJ, et al: A set of consensus mammalian mediator subunits identified by multidimensional protein identification technology. Mol Cell 14: 685-691, 2004.

11. Li XH, Fang DN and Zeng CM: Knockdown of MED19 by short hairpin RNA-mediated gene silencing inhibits pancreatic cancer cell proliferation. Cancer Biother Radiopharm 26: 495-501, 2011.

12. Shih IeM and Davidson B: Pathogenesis of ovarian cancer: clues from selected overexpressed genes. Future Oncol 5: 1641-1657, 2009. 
13. Pisano C, Bruni GS, Facchini G, et al: Treatment of recurrent epithelial ovarian cancer. Ther Clin Risk Manag 5: 421-426, 2009.

14. Nelson AE, Francis JE and Zorbas H: Population screening and early detection of ovarian cancer in asymptomatic women. Aust N Z J Obstet Gynaecol 49: 448-450, 2009.

15. Marth C, Hiebl S, Oberaigner W, et al: Influence of department volume on survival for ovarian cancer: results from a prospective quality assurance program of the Austrian Association for Gynecologic Oncology. Int J Gynecol Cancer 19: 94-102, 2009.

16. Sato S, Tomomori-Sato C, Banks CA, et al: Identification of mammalian Mediator subunits with similarities to yeast Mediator subunits Srb5, Srb6, Med11, and Rox3. J Biol Chem 278: 15123-15127, 2003.
17. Martinez LA, Naguibneva I, Lehrmann H, et al: Synthetic small inhibiting RNAs: efficient tools to inactivate oncogenic mutations and restore p53 pathways. Proc Natl Acad Sci USA 99: 14849-14854, 2002.

18. Rubinson DA, Dillon CP, Kwiatkowski AV, et al: A lentivirusbased system to functionally silence genes in primary mammalian cells, stem cells and transgenic mice by RNA interference. Nat Genet 33: 401-406, 2003.

19. Brummelkamp TR, Bernards R and Agami R: Stable suppression of tumorigenicity by virus-mediated RNA interference. Cancer Cell 2: 243-247, 2002.

20. Crnkovic-Mertens I, Hoppe-Seyler F and Butz K: Induction of apoptosis in tumor cells by siRNA-mediated silencing of the livin/ML-IAP/KIAP gene. Oncogene 22: 8330-8336, 2003. 